\title{
Three-dimensional reconstruction of stenosed coronary artery segments with assessment of the flow impedance
}

\author{
A.A. Hulzebosch ${ }^{1,3}$, C.H. Slump ${ }^{1,4}$ \& M.A. Viergever ${ }^{2,3}$ \\ ${ }^{1}$ Philips Medical Systems, productgroup Image Handling, Best, The Netherlands; \\ ${ }^{2}$ Dept. of Applied Mathematics, Delft University of Technology, Delft, The Netherlands; \\ ${ }^{3}$ present address: University of Utrecht, Dept. of Radiology \& Nuclear Medicine, Heidelberglaan 100, \\ 3584 CX Utrecht, The Netherlands; ${ }^{4}$ present address: University of Twente, Dept. Electrical Engineering, lab. \\ for Network Theory, P.O. Box 217, 7500 AE Enschede, The Netherlands
}

Key words: 3D reconstruction, 3D display, flow resistance, coronary arteriography, biplane acquisition

\begin{abstract}
In this paper preliminary results of a study about the diagnostic benefits of $3 \mathrm{D}$ visualization and quantitation of stenosed coronary artery segments are presented. As is well known, even biplane angiographic images do not provide enough information for binary reconstruction. Therefore, a priori information about the slice to be reconstructed must be incorporated into the reconstruction algorithm. One approach is to assume a circular cross-section of the coronary artery. Hence, the diameter is estimated from the contours of the vessels in both projections. Another approach is to search for a solution of the reconstruction problem close to the previously reconstructed adjacent slice. In this paper we follow the first method based on contour information. The reconstructed coronary segment is visualized in three dimensions. Based on the obtained geometry of the obstruction the pertinent blood flow impedance is estimated on the basis of fluid dynamic principles. The results of applying the reconstruction algorithms to clinical coronary biplane exposures are presented with an indication of the assessed flow impedance.
\end{abstract}

\section{Introduction}

Coronary arteriography is a standard diagnostic procedure in cardiology. With this imaging technique, however, the assessment of the severity and clinical relevance of a stenosis in a coronary artery requires much skill. Even with biplane imaging where two orthogonal views of the stenosed vessel segment are acquired, the interpretation is complicated.

This paper reports about a part of a larger study devoted to the question of whether quantification and $3 \mathrm{D}$ visualization of a stenosed artery will benefit the cardiologist in the diagnostic task mentioned above. A good overview about the quantitative assessment of stenosed coronary arteries can be found in Reiber et al. [1].

This paper is in line with previous work $[2,3,4]$ based on digitized cine-film images. In this previous work the ill-posed problem of binary $3 \mathrm{D}$ reconstruction from biplane exposures is regularized by incorporating into the reconstruction algorithm $a$ priori information, such as the shape of the adjacent (previously reconstructed) slice. The results of this paper, however, are obtained using the assumption that the cross-sections of the vessels are circular. The main topics in this paper are the reconstruction and visualization of the vessels and the assessment of the flow impedance based on the reconstructed geometry. Results of applying the 


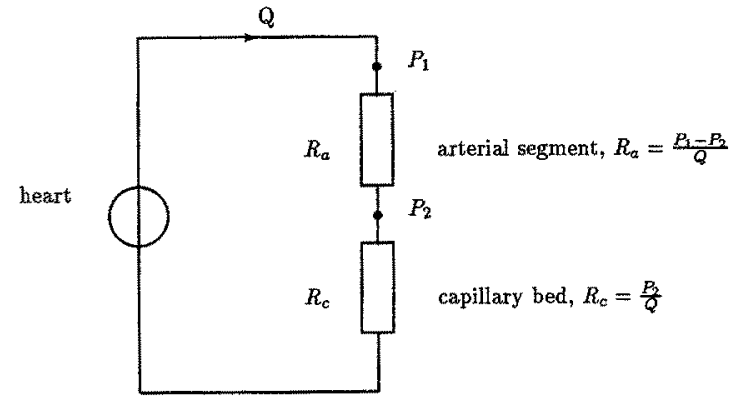

Fig. 1. Simplified coronary flow model.

developed algorithms to clinical coronary biplane exposures are presented.

When the 3D geometry of a stenosis is known, the resulting flow can be calculated in principle by means of a fluid dynamical computation. However, the computational complexity of this inverse prob- lem is prohibitive. The vascular geometry together with the boundary conditions are not known, as only recordings of flow patterns of injected contrast material are available.

We therefore seek a more modest approach and aim at the assessment of the flow impedance on the basis of a geometrical model of the shape of the stenosed artery segment (Section 3).

In this geometrical model the stenosed coronary artery consists of a set of cylindrical tubes, which is consistent with our assumption of circular vessel cross-sections. The question about the clinical relevance of the stenosis can now be addressed using a simplified hemodynamic flow model, following Logan [5]. The flow model is depicted in Fig. 1. The pulsatile character of the coronary flow is neglected. The parameters in the model are $R_{a}$, the resistance to flow in the stenosed artery segment; $R_{c}$, the

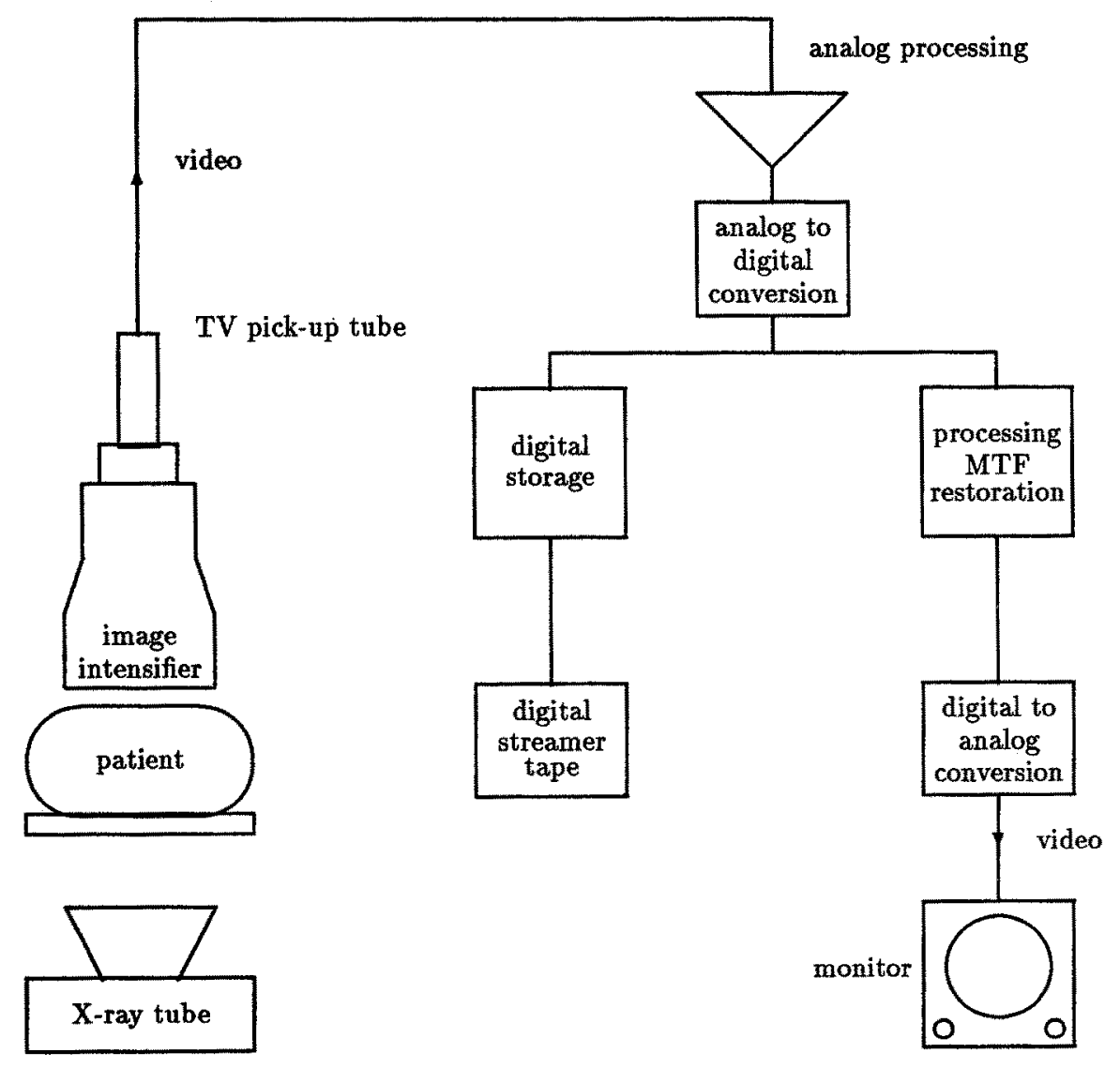

Fig. 2. Block diagram of Digital Cardiac Imaging (DCI), frontal acquisition. 


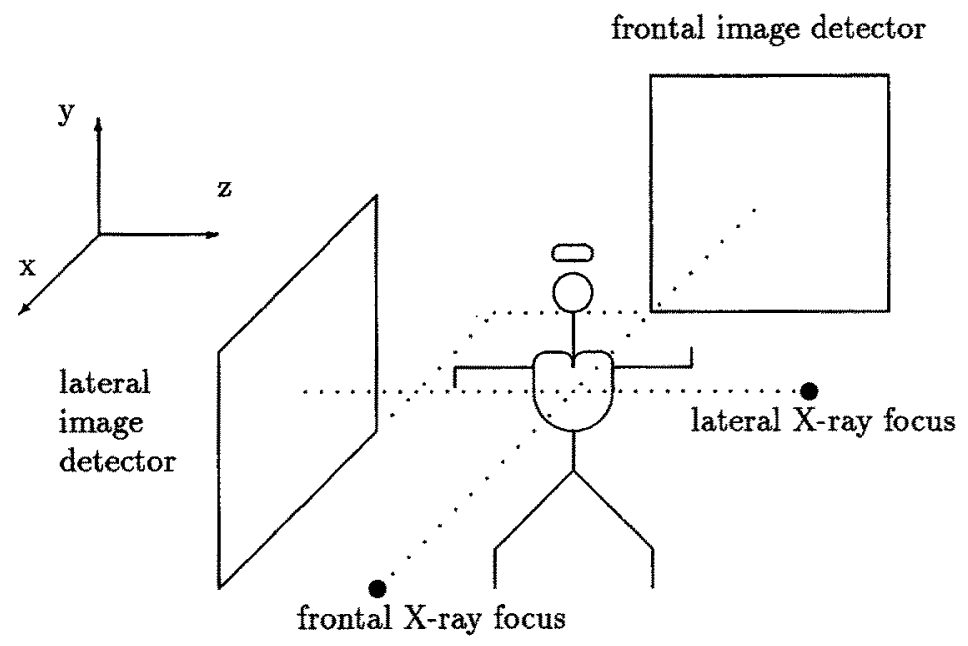

Fig. 3. The projection geometry and the coordinate frame.

resistance of the capillary bed and $P_{1}$, the mean aortic diastolic pressure. The flow $Q$ is given by $Q=P_{1} /\left(R_{a}+R_{c}\right)$. The flow $Q$ is regulated by the resistance $R_{c}$ of the capillary vessels (coronary autoregulation). Only in case of minimal $R_{c}$, the resistance $R_{a}$ of the pertinent coronary artery segment becomes flow limiting. In Section 2 the image acquisition and data handling procedures are described. The reconstruction, display and flow assessment are described in the subsequent sections.

\section{Image acquisition and data handling}

The data processed in this paper are acquired with a Philips Digital Cardiac Imaging (DCI) system which is the all digital catheterization laboratory.

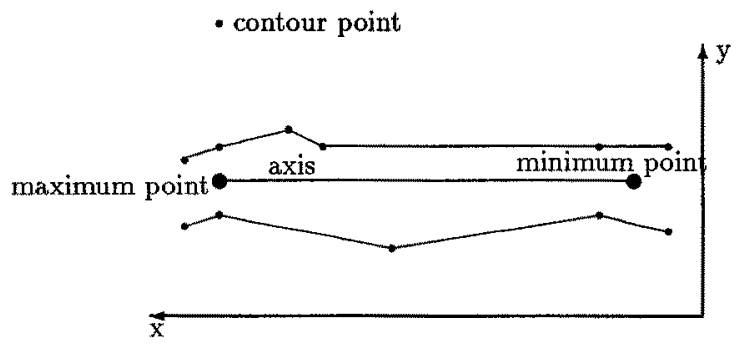

Fig. 4. The vessel axis and the contour points (lateral projection).
In fact the excellent image quality of the system together with the access to biplane images from Leiden University Hospital made us decide to look again at the problem of 3D reconstruction and display of stenosed segments of coronary vessels.

The system is schematically depicted in Fig. 2 (only the frontal image acquisition is indicated). $X$-ray pulses are generated (typ. $5-8 \mathrm{~ms}$ ) at a rate of 15-30 frames/s in order to freeze the motion of the coronary arteries which are injected with a contrast agent by means of a positioned catheter. The X-ray photons which have traversed the patient are converted into a video image by the image intensifierTV system. The resulting video signal is digitized in 8 bits after analog processing. The images are stored on a real time digital disk in $512 \star 512 \star 8$ bit up to $60 \mathrm{frames} / \mathrm{s}$ or in case of biplane up to $2 \star 30$ frames/s.

Parallel to the storage on disk, the images are processed (i.e. MTF corrected) and displayed on a monitor. A versatile user-interface is provided for viewing and post-processing.

For our purpose selected biplane runs from Leiden University Hospital were transferred from the disk and archived on a digital streamer tape in an off-line process. The tapes were transferred to the VICOM image processor of the Philips Predevelopment Group for further off-line processing.

In principle, densitometric analysis of angio- 
- translated and

rotated contour point

o interpolated contour point

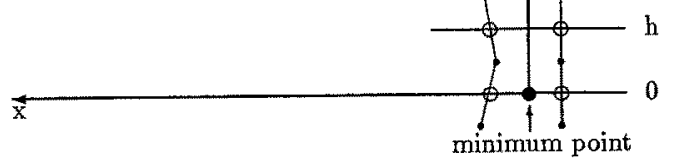

Fig. 5. The translation, rotation and interpolation of contour points.

grams requires corrections to be applied to the images. A not exhaustive list includes removal of background structures by log-subtraction of an empty mask image acquired prior to iodine injection (a cumbersome procedure in cardiac imaging due to object movements), correction for pincushion distortion due to the curved detector screen of the image intensifier, restoration of the effects of X-ray scatter and veiling glare. Algorithms with good results in clinical situations are available. However, in the feasibility phase of our project no corrections were applied as we only need to use the contour information of the imaged vessels. The images were selected such that the stenosed vessel segments were positioned not too far from the center of the image and furthermore with the frontal and lateral geometrical magnifications approximately equal.

The data handling is as follows. In the selected images the contours of the stenosed vessel segments were traced manually with a cursor under

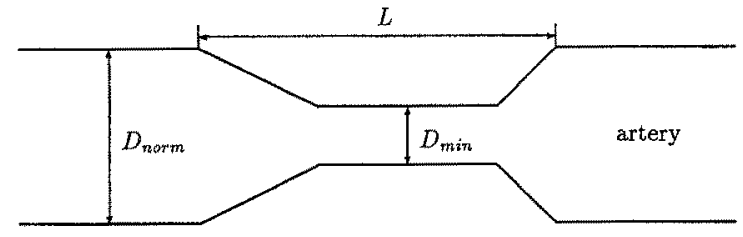

Fig. 7. Simplified geometrical model of a stenosed arterial seg. ment.

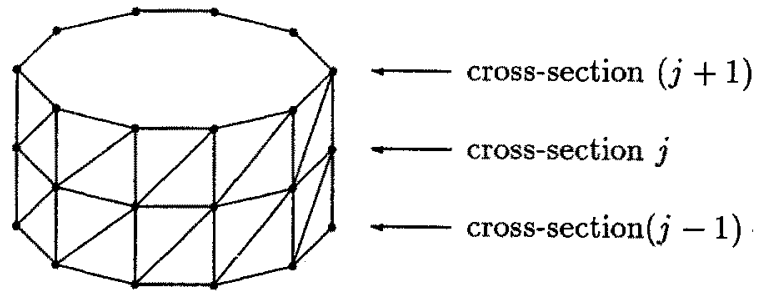

Fig. 6. Simplified drawing of the triangles which build the surface.

track-ball control. The selected contour points were stored in a file, and transferred to a VAX $11 / 750$ computer where they serve as input data for the $3 \mathrm{D}$ reconstruction and display procedure. The next section describes this procedure in greater detail.

\section{3D Reconstruction and display from circular cross-sections}

Orthogonal biplane images which are almost simultaneously acquired, are used to reconstruct coronary sections. In both projections the contours of the vessel were defined, as described in the previous section. The diameter and the location of the center of each circular cross section had to be determined from this information. The axis of the vessel segment, therefore, should not be parallel to one of the X-ray beams. In practice, the angle must be

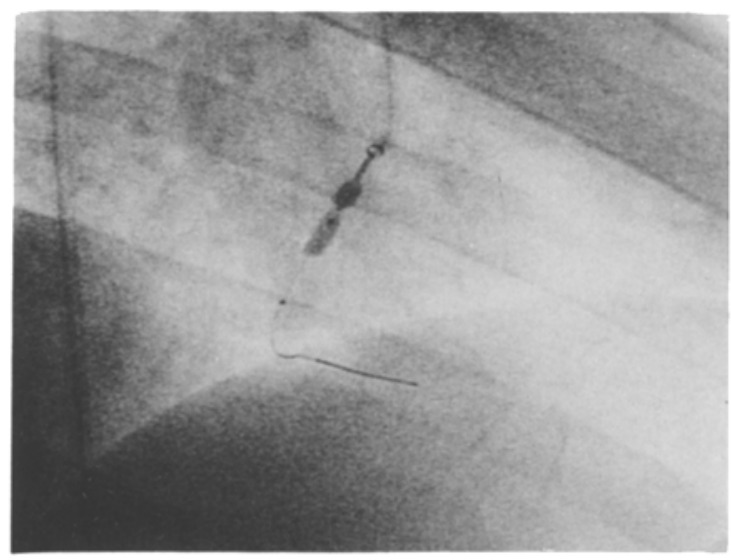

Fig. 8. Frontal angiogram first example. 


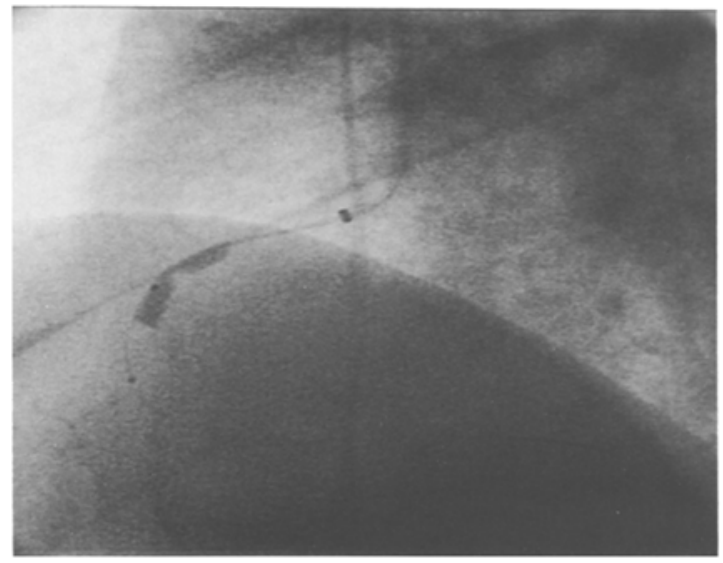

Fig. 9. Lateral angiogram first example.

more than 10 degrees with the xz-plane (see the projection geometry as indicated in Fig. 3). Note the left orientation of the coordinate frame, which is often used in 3D computer graphics.

Furthermore, it is necessary that a so-called reference point can be distinguished in both projection images. Such a marker, for example a catheter tip, is required for a good matching of the two sets of contour points. The marker was indicated by the cursor under track-ball control and its coordinates are stored.

In the first ('first' can be either frontal or lateral) image the vessel segment to be reconstructed was selected. The axis of this segment was defined by indicating in the image two points of which the coordinates are stored (Fig. 4)

The vessel contours were traced manually by a cursor under track-ball control. The contour was approximated by straight-line segments between selected contour points. The next step is indicated in Fig. 5. The points defining the vessel axis and the

Table 1. The geometrical parameters of the first stenosed arterial segment.

\begin{tabular}{lc}
\hline Length of the obstruction $L(\mathrm{~mm})$ & 5.5 \\
Minimum diameter $D_{m i n}(\mathrm{~mm})$ & 1.2 \\
Normal diameter $D_{\text {nom }}(\mathrm{mm})$ & 3.2 \\
Percentage diameter reduction $D_{\text {red }}(\%)$ & 63 \\
Percentage area reduction $A_{\text {red }}(\%)$ & 89 \\
Normal flow impedance $\times 10^{8}\left(\mathrm{~kg} \mathrm{~m}^{-4} \mathrm{~s}^{-1}\right)$ & 0.07 \\
\hline
\end{tabular}

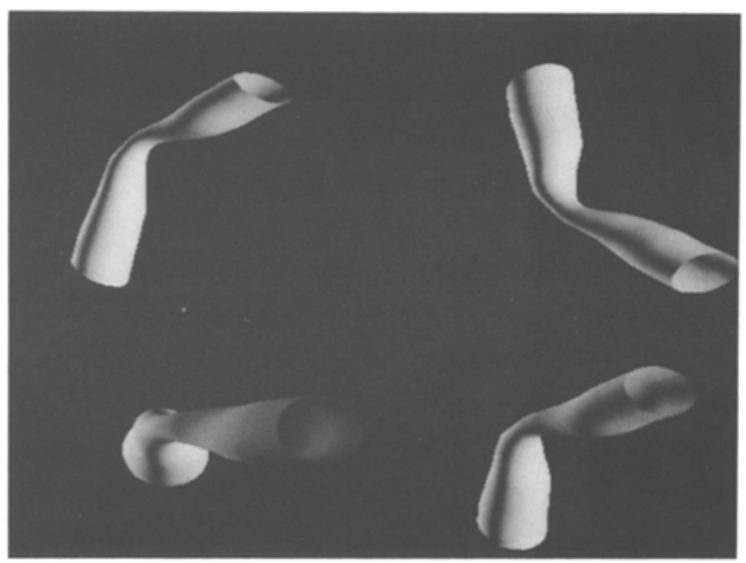

Fig. 10. Four reconstructed views of the stenosed arterial segment of Figs 8 and 9.

set of contour points were translated and rotated in such a way that the vessel axis and the minimum point coincide with the $y$-axis and the origin, respectively.

From the coordinates of the reference point the corresponding part of the vessel in the second projection image was selected. Again, two points were indicated for defining the axis of the vessel segment and the contour of the second projection was traced.

After translation and rotation, the length of the segment axis in the second projection was made equal to the length of the axis in the first projection. With linear interpolation the coordinates of the contour points at $y=k h$ were determined in both images $(k=0,1, \ldots, n)$ with $h$ the $y$-distance of the segment of the vessel to be reconstructed divided by $n$, the number of slices selected. For the $3 \mathrm{D}$ reconstruction the coordinates of the center points and the diameter of each circular slice were deter-

Table 2. The calculated flow impedance estimates for the obstruction of Fig. 10.

\begin{tabular}{lll}
\hline$Q\left(m l s^{-1}\right)$ & $R_{a}\left(10^{8} \mathrm{~kg} \mathrm{~m}^{-4} \mathrm{~s}^{-11}\right)$ & $S_{\text {red }}(\%)$ \\
\hline 0.5 & 4.8 & 98.5 \\
1.0 & 6.3 & 98.9 \\
1.5 & 7.8 & 99.1 \\
2.0 & 9.3 & 99.2 \\
\hline
\end{tabular}




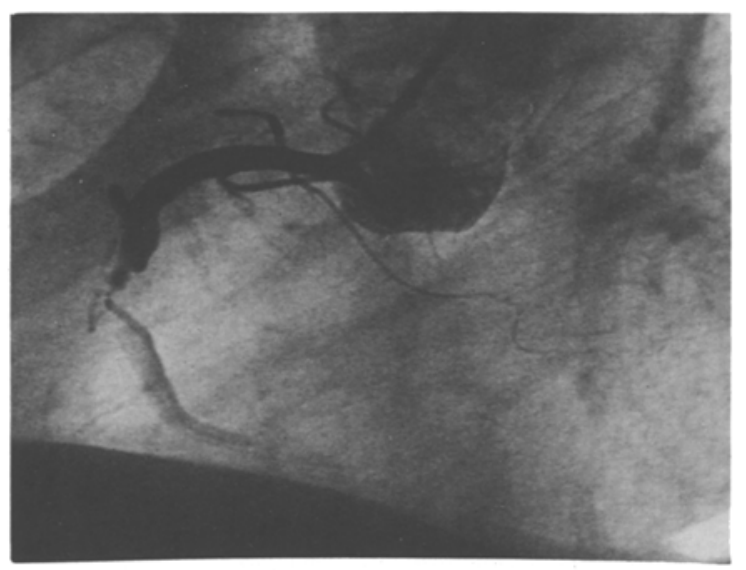

Fig. 11. Frontal angiogram second example.

mined. The diameter of the circular vessel element is defined as the mean value of the distance between the left and right contour point in both projections. Finally, the center points were rotated to the original position in the xyz-frame. The object at this stage was represented as a set of parallel circles with in principle variable radius, stacked in 3D space.

For the display of the reconstructed coronary vessel segment the coordinates of the surface points were required. Therefore, the circle elements were approximated by polygons. By generating triangles between adjacent polygon-shaped slices, the surface of the reconstructed vessel segment was built (Fig. 6) for simplicity the slices in this figure are situated right on top of each other and have the same size. For objects with circular cross-sections the tiling of the surface is quite simple by taking the same number of vertices for each polygon.

Finally, the 3D display is completed by specify-

Table 3. The geometrical parameters of the second stenosed arterial segment.

\begin{tabular}{lc}
\hline Length of the obstruction $L(\mathrm{~mm})$ & 15.0 \\
Minimum diameter $D_{\min }(\mathrm{mm})$ & 1.0 \\
Normal diameter $D_{\text {norm }}(\mathrm{mm})$ & 4.1 \\
Percentage diameter reduction $D_{\text {red }}(\%)$ & 75 \\
Percentage area reduction $A_{\text {red }}(\%)$ & 94 \\
Normal flow impedance $\times 10^{8}\left(\mathrm{~kg} \mathrm{~m}^{-4} \mathrm{~s}^{-1}\right)$ & 0.06 \\
\hline
\end{tabular}

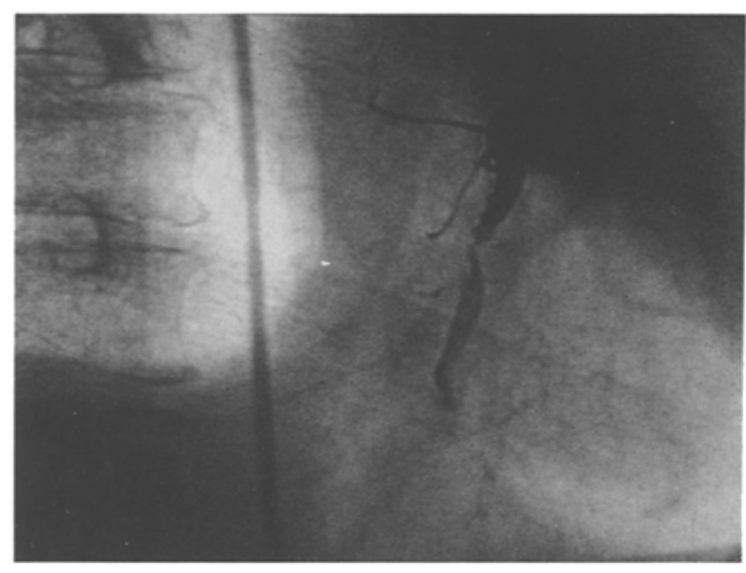

Fig. 12. Lateral angiogram second example.

ing the position of a light source and a viewing angle in the xyz-frame. Hence, the light intensities reflected from the triangle surfaces were calculated and displayed as an image. The reflected intensities were interpolated according to the Phong-shading algorithm, which results in smooth looking objects (see the results in Section 5).

\section{Flow impedance}

As already stated in the introduction the flow impedance of the reconstructed vessel segment can be computed on the basis of a geometrical model of the stenosed coronary artery. Because we neglected here the pulsatile character of the coronary flow, the imaginary part of the flow impedance equals zero. In Fig. 7 the model is presented; the following parameters are indicated explicitly: the minimum and normal diameters $D_{\min }$ and $D_{\text {norm }}$ respectively and the length of the obstruction $L$.

Table 4. The calculated flow impedance estimates for the obstruction of Fig. 13.

\begin{tabular}{lll}
\hline$Q\left(m l s^{-1}\right)$ & $R_{a}\left(10^{8} \mathrm{~kg} \mathrm{~m}^{-4} \mathrm{~s}^{-1}\right)$ & $S_{\text {red }}(\%)$ \\
\hline 0.5 & 21.6 & 99.72 \\
1.0 & 25.2 & 99.76 \\
1.5 & 28.8 & 99.79 \\
2.0 & 32.4 & 99.81 \\
\hline
\end{tabular}




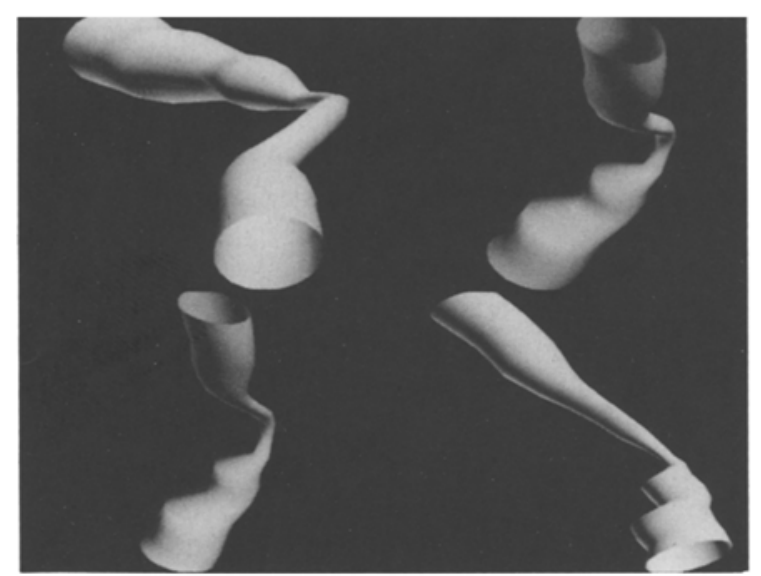

Fig. 13. Four reconstructed views of the stenosed arterial segment of Figs 11 and 12.

In vitro experiments of Lipscomb and Hooten [8] and Seeley and Young [9] have revealed that the other geometrical parameters of Fig. 7, such as eccentricity and exit angle, are of limited importance. Experiments by van Dreumel [10] have shown that the influence of the exit angle is negligible for Reynold numbers larger than 50 , a condition certainly valid in coronary vessels. In order to achieve an assessment of the flow impedance of an obstruction we calculated the three geometrical parameters $D_{m i n}, D_{n o r m}$ and $L$. From these parameters the flow resistance $R_{a}$ was computed as:

$$
R_{a}=\frac{8 \pi \mu L}{A_{\min }^{2}}+\frac{\varrho}{2}\left(\frac{1}{A_{\min }}-\frac{1}{A_{\text {norm }}}\right)^{2} Q
$$

with

$$
\begin{array}{ll}
L & =\text { length of the coronary obstruction } \\
\mu & =\text { blood viscosity }\left(3 \times 10^{-3} \mathrm{~kg} \mathrm{~m}^{-1} \mathrm{~s}^{-1}\right) \\
Q & =\text { blood density }\left(1 \times 10^{3} \mathrm{~kg} \mathrm{~m}^{-3}\right) \\
Q & =\text { mean coronary blood flow } \\
A_{\text {min }} & =\text { minimum vessel cross-section } \\
A_{n o r m} & =\text { normal vessel cross-section }
\end{array}
$$

This formula for the calculation of the flow resistance is widely used in the literature, see Young and Tsai [6, 7], Gould [11], Reiber et al. [1]. The first term in equation (1) equals the Hagen-Poisseuille pressure drop over a rigid pipe (cross-section $A_{\text {min }}$ ) caused by the viscosity of a laminarly flowing New-

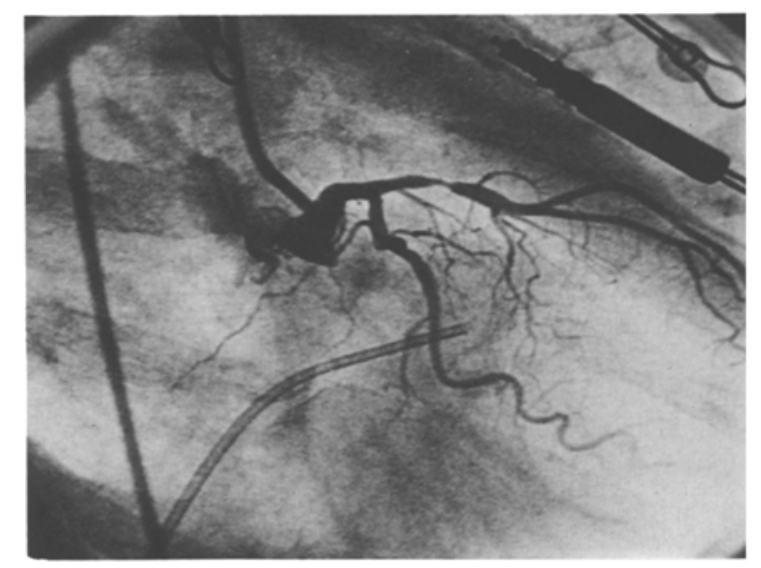

Fig. 14. Frontal angiogram third example.

tonian fluid. The second term indicates the pressure drop due to separation at the end of the stenosis. Note that this term is proportional to the mean coronary blood flow $Q$. The published agreement between theory and experiment related to equation (1) (the error is about 15\%) is sufficient for the purpose of our study. In order to evaluate the obtained flow impedance values, we calculated a parameter which we termed the "percentage flow conductivity reduction'. This parameter is defined as:

$$
S_{r e d}=\left(1-\frac{R_{n o r m}}{R_{a}}\right) \cdot 100 \%
$$

with $R_{a}$ the flow resistance of eq. (1) and $R_{\text {norm }}$ the Hagen-Poisseuille resistence of the unconstricted vessel which follows from (1) by substitution of $A_{\text {norm }}$ for $A_{\text {min }}$. We also calculated the widely used parameters percentage diameter and percentage area reduction ( $D_{r e d}$ and $A_{r e d}$, respectively), which are defined as follows:

$$
\begin{aligned}
& D_{\text {red }}=\left(1-\frac{D_{\text {min }}}{D_{\text {norm }}}\right) \cdot 100 \% \\
& A_{\text {red }}=\left(1-\frac{A_{\text {min }}}{A_{\text {norm }}}\right) \cdot 100 \%
\end{aligned}
$$

\section{Results}

In this section the theory of this paper is applied to 


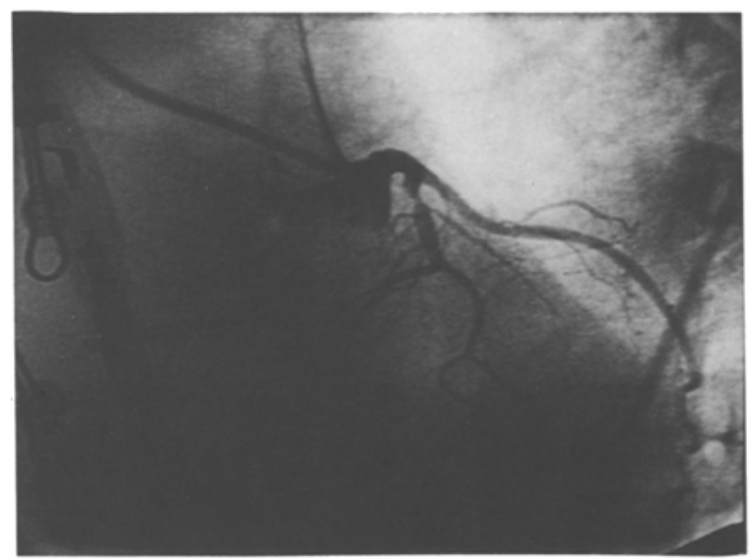

Fig. 15. Lateral angiogram third example.

three coronary arterial segments. Of each stenosed artery segment the frontal and lateral coronary angiograms are given, followed by 3D visualizations of the pertinent segment under four different viewing angles.

Subsequently, the flow impedance values were calculated on the basis of the reconstructed geometry. The geometrical parameters were determined in pixels. From the known size of a catheter the calibration factor was calculated in $\mathrm{mm}$ /pixel. The frontal and lateral coronary angiograms of the first example are presented in Figs 8 and 9, respectively. The imaged object is a balloon, partially inflated with contrast agent, following the shape of the stenosis. The reconstructed result is presented for four different viewing angles in Fig. 10. The obtained geometrical parameters are summarized in Table 1.

The calculated flow impedance values as a function of the mean coronary blood flow $Q$ are given in Table 2.

Table 5. The geometrical parameters of the third stenosed arterial segment.

\begin{tabular}{lc}
\hline Length of the obstruction $L(\mathrm{~mm})$ & 12.0 \\
Minimum diameter $D_{\min }(\mathrm{mm})$ & 1.2 \\
Normal diameter $D_{\text {norm }}(\mathrm{mm})$ & 3.9 \\
Percentage diameter reduction $D_{\text {red }}(\%)$ & 68 \\
Percentage area reduction $A_{\text {red }}(\%)$ & 90 \\
Normal flow impedance $\times 10^{8}\left(\mathrm{~kg} \mathrm{~m}^{-4} \mathrm{~s}^{-1}\right)$ & 0.07
\end{tabular}

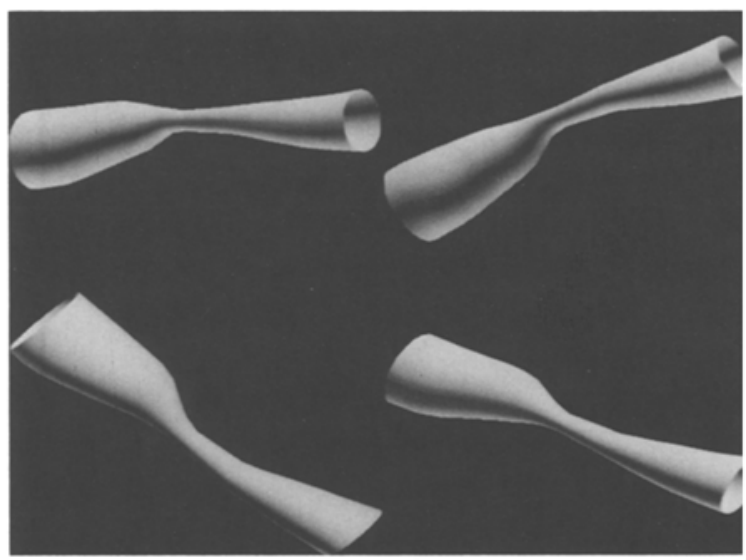

Fig. 16. Four reconstructed views of the stenosed arterial segment of Figs 14 and 15.

The frontal and lateral coronary angiograms of the second example are presented in Figs 11 and 12, respectively. The reconstructed results for four different viewing angles are displayed in Fig. 13. The obtained geometrical parameters are summarized in Table 3.

The calculated flow impedance values as a function of $Q$ are given in Table 4. The frontal and lateral coronary angiograms of the third example are presented in Figs 14 and 15, respectively. The reconstructed result is presented for four different viewing angles in Fig. 16. The obtained geometrical parameters are summarized in Table 5, while the calculated flow impedance values as a function of $Q$ are given in Table 6.

\section{Discussion of the results}

The few processed cases do not justify a firm con-

Table 6. The calculated flow impedance estimates for the obstruction of Fig. 16.

\begin{tabular}{lll}
\hline$Q\left(m l s^{-1}\right)$ & $R_{a}\left(10^{8} k g ~ m^{-4} s^{-1}\right)$ & $S_{r e d}(\%)$ \\
\hline 0.5 & 8.7 & 99.2 \\
1.0 & 10.3 & 99.3 \\
1.5 & 11.9 & 99.4 \\
2.0 & 13.5 & 99.5 \\
\hline
\end{tabular}


clusion. Of the three cases presented, the stenosis of the second study is judged the most severe, followed by study three and one, respectively. The three measures for indicating the severity of a coronary stenosis $A_{\text {red }}, D_{\text {red }}$ and $S_{\text {red }}$ are consistent. As only $S_{r e d}$ depends on the blood flow $Q$, measured flow values e.g. by means of Doppler flow catheters, can reveal more about the clinical relevance of the three measures. Note that for very small flow values $S_{\text {red }}$ becomes equal to $D_{\text {red }}$ as can be easily seen from the definitions (2), (3).

Another relative straight-forward extention of the presented work would take the pulsatile character of the coronary flow into account. This leads to complex flow impedance values.

The responses of a few selected cardiologists when confronted with the examples presented in the previous section were promising/mitigated enthousiasm. The off-line character of the processing was clearly felt as a severe drawback. The $3 \mathrm{D}$ images were not presented to the cardiologist during a catheterization procedure but only weeks after the examination or treatment of the patient. As a result, the diagnostic value added was very little (if at all).

Our conjecture is that $3 \mathrm{D}$ visualization and quantification of the flow impedance of the stenosis are useful diagnostic tools, provided the computations can be carried out on-line between cardiac exposure runs.

It is evident, that further research in this direction must be carried out.

\section{Acknowledgements}

We like to thank Prof. dr. B. Buis and co-workers of the Cardiology Department, University Hospital Leiden, The Netherlands and Mr. A.C. van Benthem, Application Department, Philips Medical Systems, Best, The Netherlands for providing us with the clinical coronary biplane images. The 3D display program is a courtesy of dr. I.C. Carlsen of Philips Forschungs Labor Hamburg (PFH), Fed- eral Republic Germany. The help of Mr. H. Stegehuis M.Sc. in making the hardcopies of the images presented in this paper is gratefully acknowledged.

\section{References}

1. Reiber JHC, Serruys PW, Slager CJ. Quantitative coronary and left ventricular cineangiography. Martinus Nijhoff Publishers, Dordrecht, The Netherlands, 1986.

2. Slump CH, Gerbrands JJ. A network flow approach to reconstruction of the left ventricle from two projections. Comput Graph Image Process 1982; 18: 18-36.

3. Reiber $\mathrm{JHC}$ et al. 3D reconstruction of coronary arterial segments from two projections. Digital Imaging in Cardiovascular Radiology. In: Heintzen PH, Brennecke R, (eds). Thieme, Stuttgart, 1983; 151-63.

4. Gerbrands JJ, Slump CH. 3D reconstruction of homogeneous objects from two Poisson-distributed projections. Pattern Recognition Letters 1985; 3; 137-45.

5. Logan SE. On the fluid mechanics of human coronary artery stenosis, IEEE Trans Biomed Eng 1975; vol BME-22: 327-34.

6. Young DF, Tsai FY. Flow characteristics in models of arterial stenosis I. Steady flow, Journal of Biomechanics 1973; 6: $396-410$.

7. Young DF, Tsai FY. Flow characteristics in models of arterial stenosis II. Unsteady flow. Journal of Biomechanics $1973 ; 6: 547-59$.

8. Lipscomb K, Hooten S. Effect of stenotic dimensions and blood flow om the hemodynamic significance of model coronary arterial stenoses. American Journal of Cardiology 1978; vol 42: 781-92.

9. Seeley BD, Young DF. Effect of geometry on pressure losses across models of arterial stenoses. Journal of Biomechanics 1976; 9: 439-48.

10. van Dreumel SC. Drukval - debiet relaties in coronaire vernauwingen (in Dutch). M.Sc. thesis. Delft University of Technology, The Netherlands, 1986.

11. Gould KL. Dynamic coronary stenosis. American Journal of Cardiology 1980; vol 45: 286-92.

Address for offprints:

C.H. Slump

University of Twente

Dept. Electrical Engineering

Lab. for Network Theory

P.O. Box 217

7500 AE Enschede

The Netherlands 